\title{
Heterogeneous Cellular Interworking of WIMAX and UMTS for Sensitive Real-Time Services
}

\author{
Mina Malekzadeh \\ Electrical and Computer Faculty of Hakim Sabzevari University Sabzevar, Iran \\ Email: m.malekzadeh@hsu.ac.ir
}

\begin{abstract}
Since internet connections are provided through a variety of disparate networks, connecting these networks and supporting heterogeneous interworking is a major issue particularly in cellular networks. The interworking issue becomes even more challenging when it comes to providing QoS for real time services. To improve QoS requirements of the real time data and to increase inter cellular mobility, we propose a costeffective framework structure consists of four separate cellular models. The framework includes a heterogeneous interwork model that integrates cellular WiMAX and UMTS. Moreover, a pure WiMAX network model along with two pure UMTS network models is set up by the framework. The performance of the heterogeneous model is evaluated via simulation and analyzed against the measured metrics of the pure models to quantify the level of improvements from QoS of the real time packets point of view. Based on the results, the recommendations are made on the most appropriate model in regard to better QoS for VoIP in cellular networks.
\end{abstract}

Index Terms-Cellular interworking, Heterogeneous networks, WiMAX, UMTS, VoIP

\section{INTRODUCTION}

Nowadays there are different types of available access networks which are used by end users to connect to the internet. Thus, the users must be provided with seamless network connectivity to stay connected while moving around from one place to another. This seamless network connectivity is achieved by connecting different types of networks which is called heterogeneous internetworking. By integrating different network technologies into one common heterogeneous network architecture, they can coexist and interoperate with each other and improve network performance in term of Quality of Service (QoS) [15].

Heterogeneous wireless networks may incorporate wireless local area networks (WLAN), wireless personal area networks (WPAN), wireless metropolitan area networks (WMAN) and wireless wide area networks (WWAN) including cellular networks and satellite [2]. Among these architectures, heterogeneous cellular interworking provides substantial ability in increasing network data rate and coverage area making it is more suitable for mobile end users. Heterogeneous cellular interworking is provided by integration of different radio access technology such as universal mobile telecommunications system (UMTS), IEEE 802.16 worldwide interoperability for microwave access (WiMAX), long term evolution (LTE), WLAN, etc.

While the combination of cellular networks provides better service to end users, it also has its own issues. The types of networks that are integrated into a heterogeneous architecture, each have been developed separately with their own specific characteristics. These special requirements of cellular networks make the interworking between them a challenging task particularly for QoSsensitive real time data.

In this work we propose a cellular framework to increase the inter-cellular mobility yet to improve QoS of multimedia data. The framework consists of four models. The first model is a heterogeneous model that integrates WiMAX [13,14] and UMTS cellular networks. Along with the heterogeneous model, the framework includes three pure models for WIMAX and UMTS networks.

The performance of the proposed heterogeneous network model is evaluated and analyzed against the measured metrics of the pure network models. Special attention is paid to the VoIP packets. By studying several cellular scenarios, the main objective of this work is to analyze various alternatives for interworking of UMTS and WiMAX networks to optimize QoS for VoIP real time data and achieve better network performance in demand for higher data rate.

The rest of this work is organized as follows. Section 2 provides the related works. Section 3 describes the design structure of the proposed framework for interworking. Section 4 presents results and discussions. The conclusion is provided in section 5 .

\section{RELATED WORKS}

Due to importance of the interworking of different types of networks, it has been attracted the attention of many researches.

WiMAX and UMTS interworking are investigated in [1] for IP Multimedia Subsystem (IMS) based networks. Two network models as Hybrid Coupled WiMAX-UMTS with and without QoS provisioning are created and compared. The two models are compared with each other however the effect of having a merged network on performance of IMS is not taken into account in their work. 
In [2] authors emphasis on importance of heterogonous networks and thereby develop a model and simulation environment to present and analyze the performance of WLAN and UMTS integration. The aim is to identify an architecture that can provide better overall performance and flexible interworking for handover. The authors mainly focus on integrating Wi-Fi and UMTS networks while the WiMAX networks are not taken into their consideration.

Heterogonous networks are also considered in [3]. By implying that the usage of wireless network is growing day by day, the authors create two heterogeneous network architectures by integrating Wi-Fi with WiMAX and also Wi-Fi with UMTS. The two architectures are simulated and the results are compared in term of a better voice quality.

Integrating WLAN with cellular networks is also discussed in [4]. The distance based path-loss model is considered in Internet Multimedia Subsystem (IMS) integrating UMTS-WiMAX-WLAN. The integrated model is used to select the efficient radio mode with expected QoS. The integration of WLAN, WIMAX and UMTS is also discussed in [5, 12].

In order to execute intersystem handover, the authors implement the algorithm of interworking architecture between WiMAX, GPRS and UMTS in [6]. However, the roaming performance is not compared beyond the internetworking on pure cellular networks.

Authors in [7] propose a framework for interworking of the UMB, WLAN and WiMAX technologies. The download and upload response time of Email along with voice jitter and packet end-to-end delay are simulated to evaluate the proposed algorithm.

The loose and tight coupling as two main interworking architectures are investigated in [8]. The authors highlight the objectives, features, and challenges of internetworking. However, no implementation is provided in order to evaluate the overall performance. A survey of interworking architectures also provided in [9, $11]$.

A handover framework is introduced in [10] for interworking of LTE, WiMAX and WLAN technologies. The objective is to offer seamless high quality IP-based multimedia services to users at any time during the handoff process.

Based on the related works, there is no existing research regarding to development of a framework capable of modeling and implementing the cellular interworking along with pure network cores to which it is compared to. This need is important as it can give a guideline to telecommunication industry for developing new techniques in demand for higher data rates. By taking this need into account, this paper contributes to the identification of the most appropriate model in regard to better QoS for VoIP in cellular networks.

\section{PROPOSED FRAMEWORK}

The structure of the proposed framework consists of four separate network models: a cellular interworking that integrates WiMAX and UMTS, a pure WiMAX network, a pure UMTS network with two independent cores and finally a pure UMTS network with one common core.

The following assumptions are set out for all the four models:

- To avoid side effects of the network complexity and to simplify the simulation models, the point to point real time traffics are exchanged between a transmitter and a receiver.

- The real time VoIP traffics are transmitted from calling Node1 to the called Node 2 in all four models in the framework.

- To provide similar network conditions for the fair comparison purpose, the calling node in all models transmits equal amount of real time traffic to the called node.

- The simulation time to run each experiment is one hour.

The first model in the framework is the heterogeneous network model. In this model, the radio access network at the calling node side is WiMAX while it is UMTS at the called node side. The access service network (ASN) comprises one cell supported by a wireless base station and one ASN gateways (ASNGW) that together form the radio access network at the calling node side. The gateway controls and aggregates the traffic from the WiMAX base station.

The radio access network at the called node side is UMTS terrestrial radio access network (UTRAN) that handles all radio related functionality. It consists of a Node B directly connected to the radio network controller (RNC) which is responsible for radio resource management and control of the Node B. To handle packet switched services by the UMTS core network, the RNC in turn is connected to serving GPRS support node (SGSN) and gateway GPRS support node (GGSN).

Finally to merge these two disparate networks and provide interworking, the GGSN and ASN gateway are connected through the internet backbone. This heterogeneous model is presented in Fig. 1.

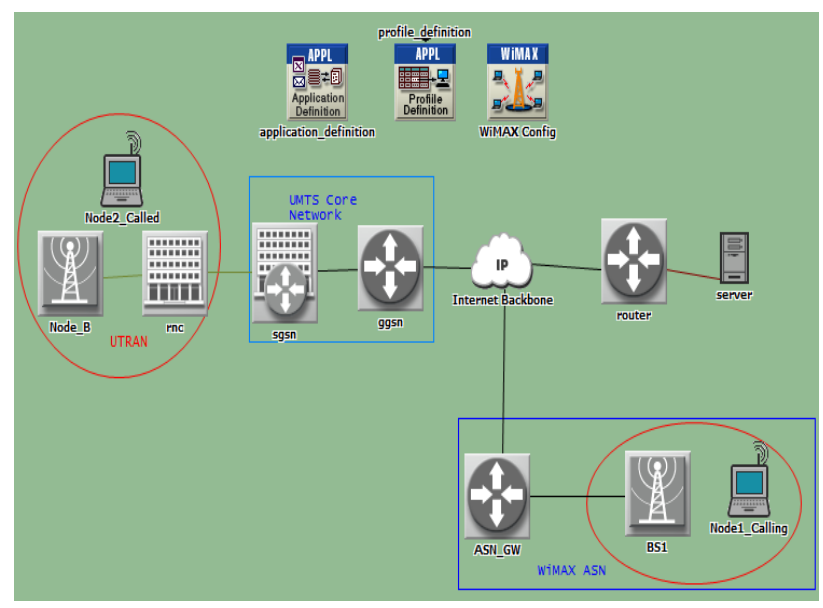

Fig.1. Heterogeneous network model 
The next model in the framework is a pure WIMAX model consists of two separate cells each of which supported by its own wireless base station. The two cells are connected to a common ASN gateway through the internet backbone. This model is presented in Fig. 2.

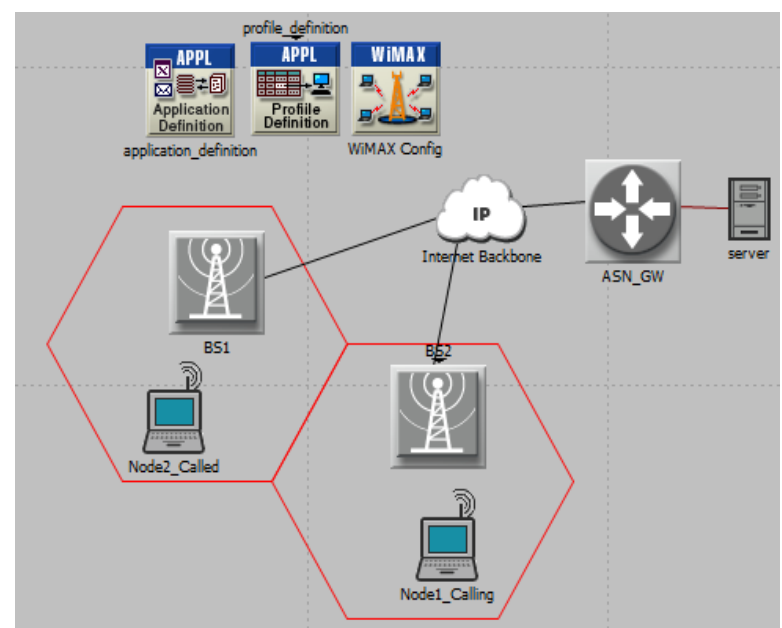

Fig.2. Pure WIMAX model

As the third and fourth model, the framework includes two pure UMTS models. In a complex pure UMTS model there may be various strategies for combination of the network components to achieve different purposes based on the particular requirements of the network. Thus by considering two different configurations for pure UMTS model, we design two separate models.

The goal of designing two different pure UMTS in the framework is to verify the parameters that can affect the network performance and also to identify which configuration can provide better QoS support. This is due to the fact that identifying pros and cons of different cellular strategies can help the network designers and operators to optimize and adopt the right models to perform specific services for that network.

The first pure UMTS model consists of two independent cores to support the called and calling nodes independently through the internet backbone. For simplicity we refer to this model as UMTS-dual which is presented in Fig. 3.

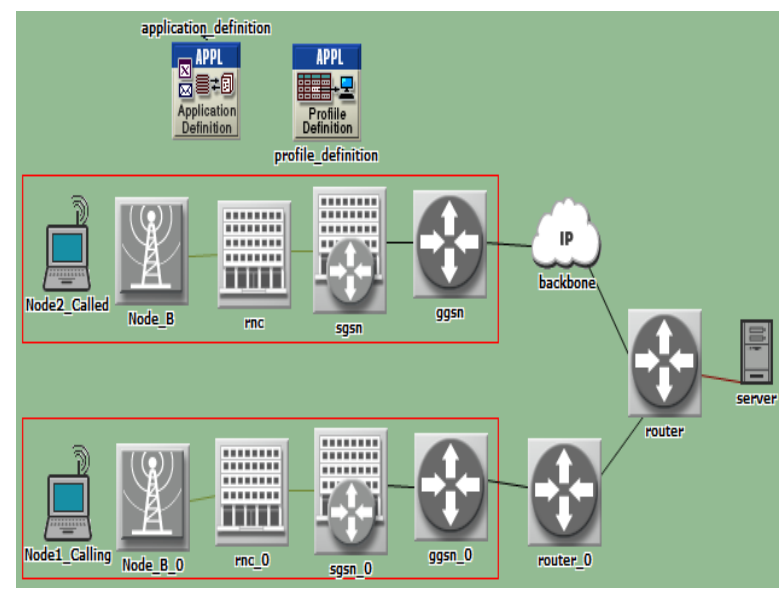

Fig.3. Pure UMTS model with two independent cores (UMTS-dual)
The second pure UMTS model is designed with reduced number of network elements in compare to the first pure UMTS model. It consists of one common core to independently support both called and calling nodes. For simplicity we refer to this model as UMTS-single which is presented in Fig. 4.

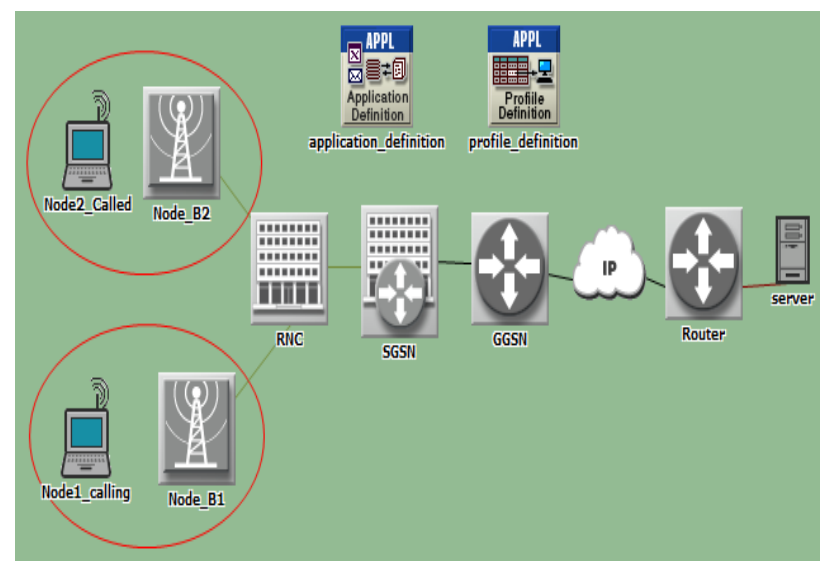

Fig.4. Pure UMTS model with a common core (UMTS-single)

The simulation models are developed using opnet14.5 simulator environment. The ability of the four models to sustain the expected QoS for real time VoIP traffics is measured independently in terms of throughput, average end-to-end delay and delay variations. Based on these metrics, a variety of experiments are simulated for each model in the framework while attention is also paid to the kind of codec used by the VoIP packets.

\section{Simulation Results}

We need to verify how the differences in the characteristics and internal structure of the four models in the framework would affect the transmission of the audio streams. Thus, we run the simulation models each of which for one hour to measure the performance metrics. This section provides the results obtained by conducting multiple scenarios and simulation runs of the proposed framework.

\section{A. Throughput of audio streams}

To measure the bit rate of the audio streams, first we measure the bytes per second of the VoIP data sent by the calling node in each model which presented in Fig. 5.

As we can see from the above results, the calling nodes load the same amount of audio data into all the four models. This similarity is necessary to provide fair comparative conditions for the models and to indicate how much of these sent data can in fact be received by the called nodes in each model. 


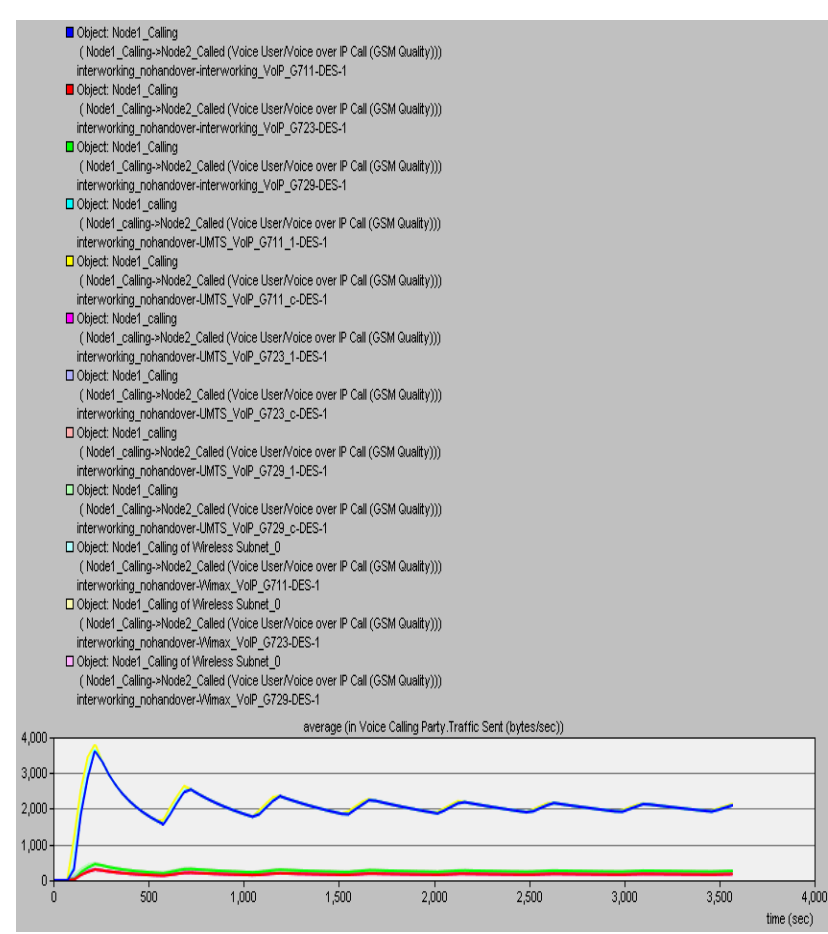

Fig.5. Overall audio stream load sent by the calling node in all four models

In other words, the comparison between the amount of sent and received audio data will describe the ability of each model to handle traffic of the same offered load. Form the above results, we also observe that the type of codec has direct impact on the amount of traffic sent from a device. To simplify the analysis, we redraw the above graph this time based on type of codec which convey a much better picture of codec impact. The results are provided in Fig. 6 A-C for G.711, G.729, and G.723 respectively.

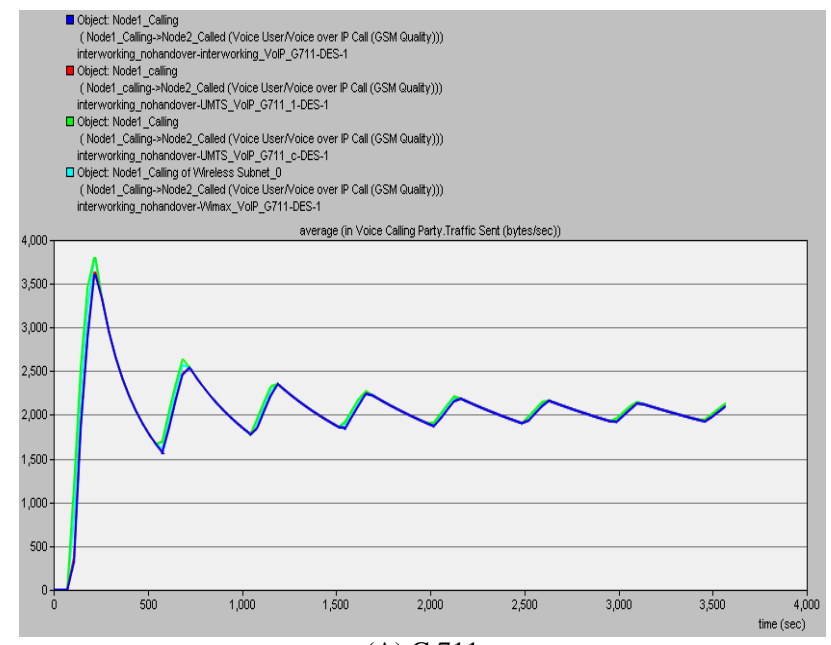

(A) G.711

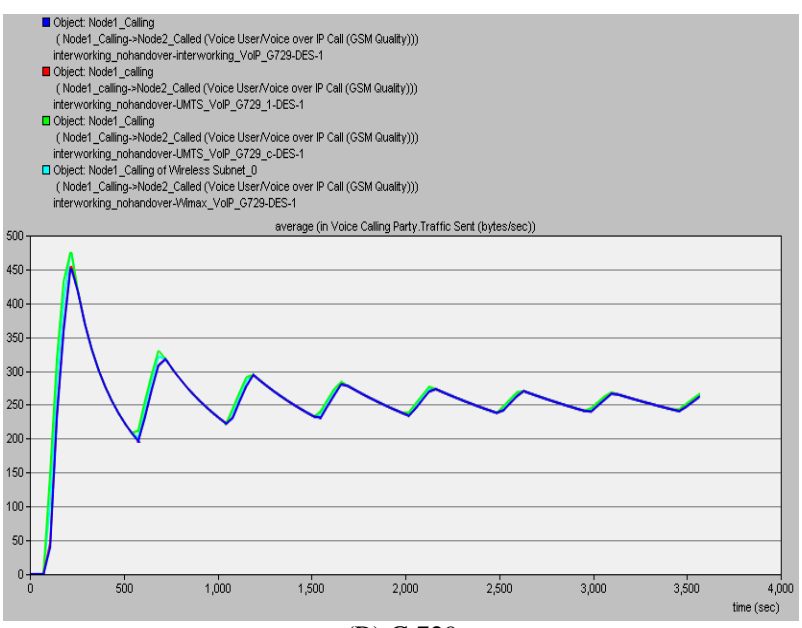

(B) G.729

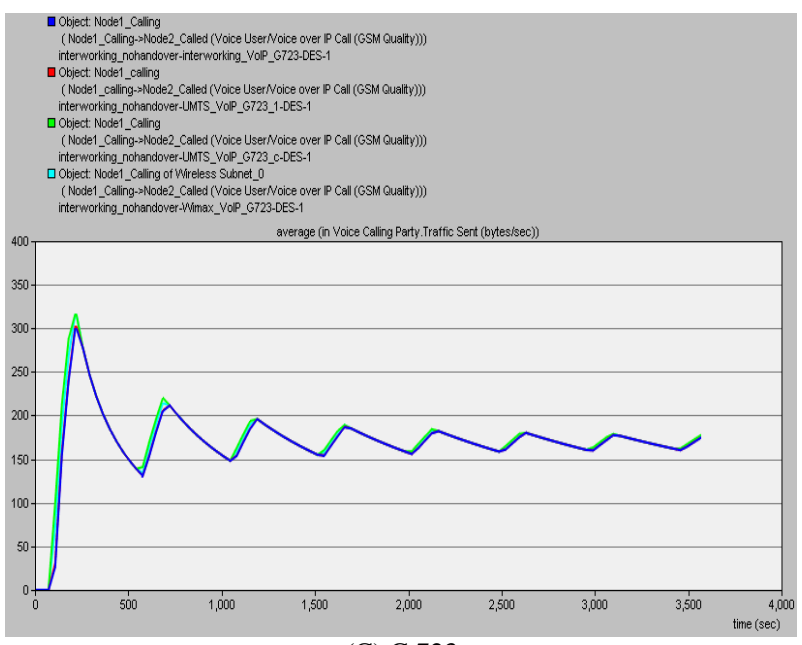

(C) G.723

Fig.6. Overall audio stream load sent by the calling node based on type of codec

As we can see more clearly now, regardless of the structure of the cellular radio access mode, the transmission traffics for G.729 and G.723 is much lower than the G.711. In this regard, there is very small difference between sent traffic in G.729 and G.723. The reason is that the G.711 unlike the G.729 and G.723 uses no compression. Typically the process of compressing and decompressing the voice on the both ends of the call is a time consuming process which imply latency to the entire voice transmission process. Also, the reason for small differences between G.729 and G.723 is that G.729 uses less compression than G.723.

Now as we mentioned, after identifying the amount of sent traffic, it is time to measure how much of these sent data are practically received by the called nodes in each model. The details of throughput measurements corresponding to the heterogonous, pure WIMAX, UMTS-single, and UMTS-dual models are provided in Fig. 7 A-D respectively. 


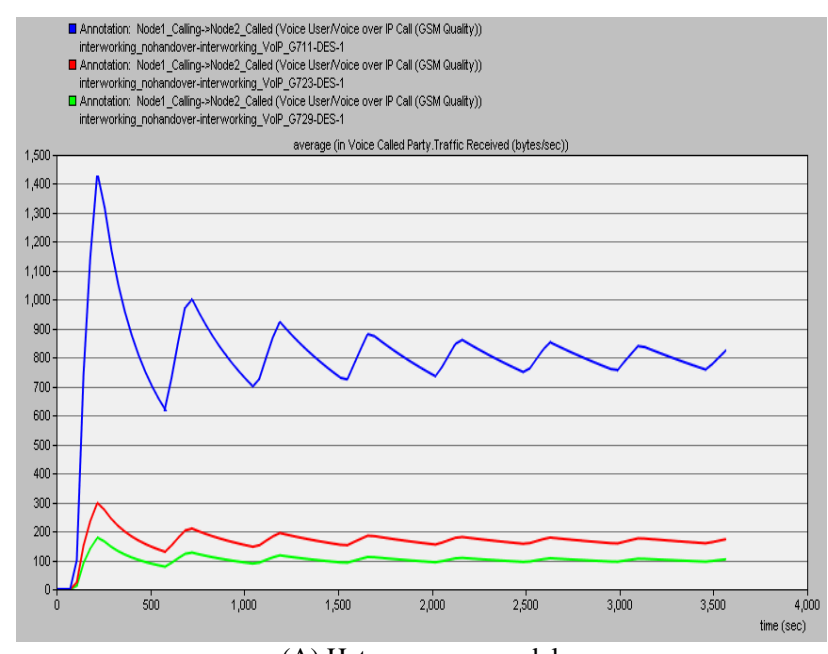

(A) Heterogonous model

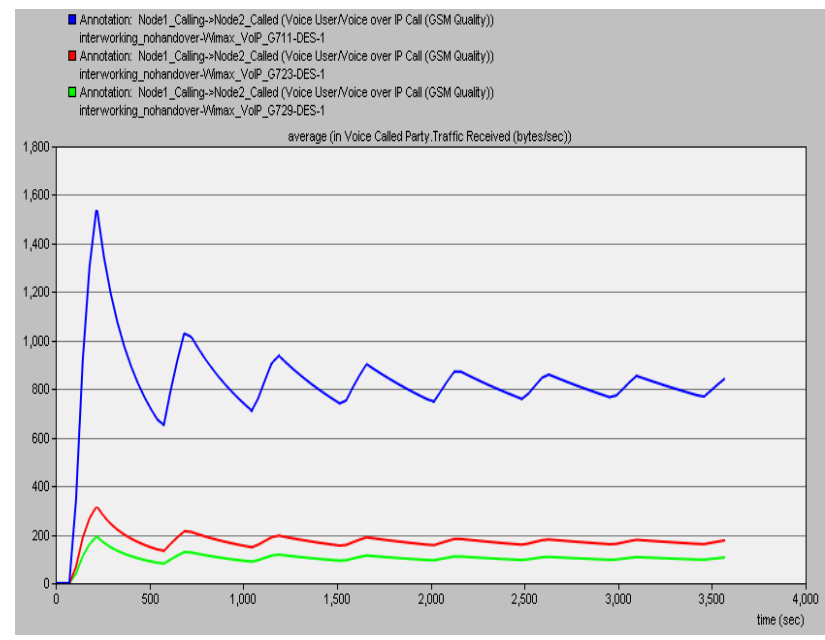

(B) Pure WIMAX model

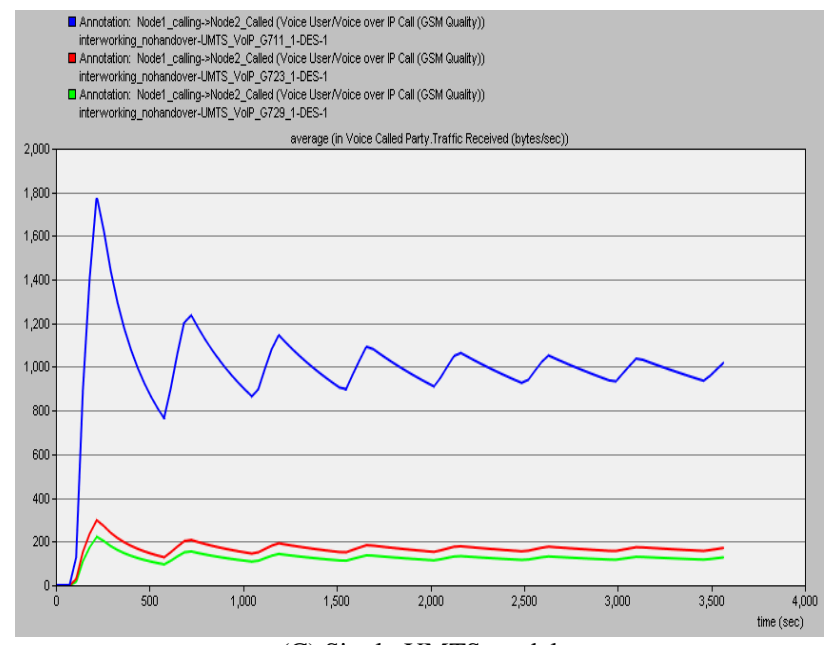

(C) Single-UMTS model

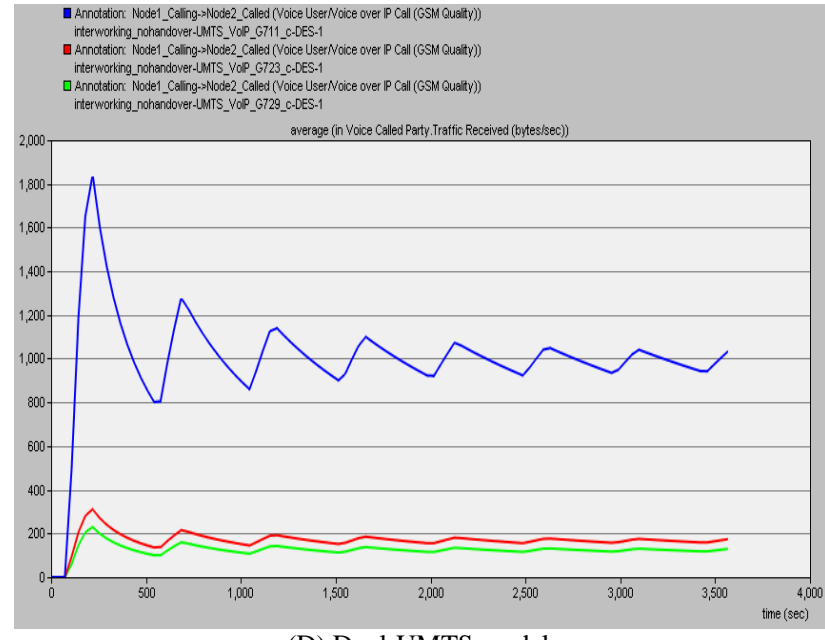

(D) Dual-UMTS model

Fig.7. Throughput measurements of the four models

From the results presented in the above figure some information are derived. As a first result we can see that regardless of the type of radio access, the amount of received traffics in all models is much less than the amount of sent traffic discussed in Figs. 5 and 6.

Moreover, the throughput rate remains at almost the same level in the four models with slight differences. The both UMTS models achieve the same throughput as each other proving that having a common core or two independent cores does not affect the throughput of the voice traffics.

By looking more into details, we see that throughput received by each called node in the UMTS models is a little higher than either heterogonous interworking model or WiMAX model. By ignoring this slight difference, the simulation results confirm that the despite the differences in the radio access networks, using the same codec, the four models do not differ significantly in regard to throughput of the voice traffics.

The simulation results also suggest that G.711 codec is beneficial for enhancing the throughput in all the models.

\section{B. Average of end-to-end delay for audio streams}

In order to study the performance stability of each model in the proposed framework, we measure the average delay experienced by voice traffics. The mean end-to-end delay results for the heterogonous, pure WIMAX, UMTS-single, and UMTS-dual models are depicted in Fig. 8 A-D respectively. 


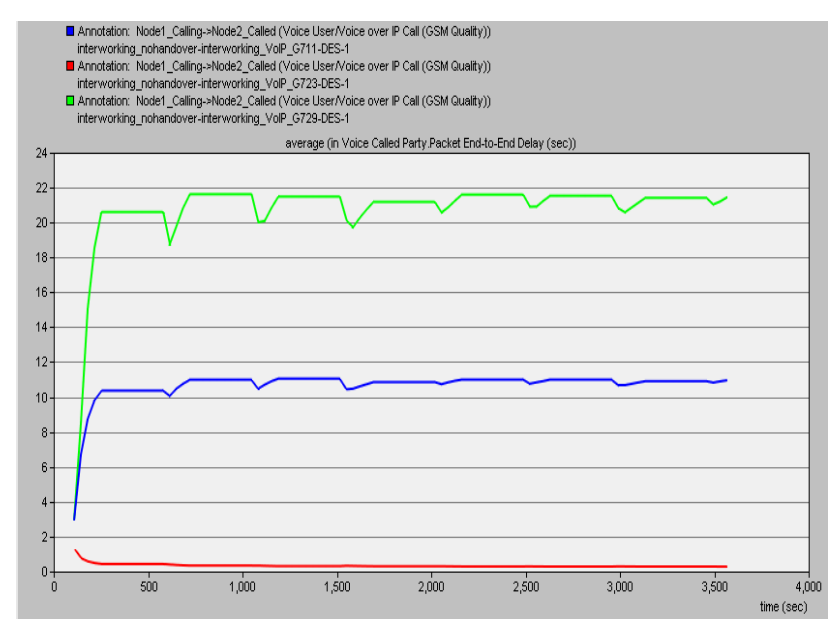

(A) Heterogonous model

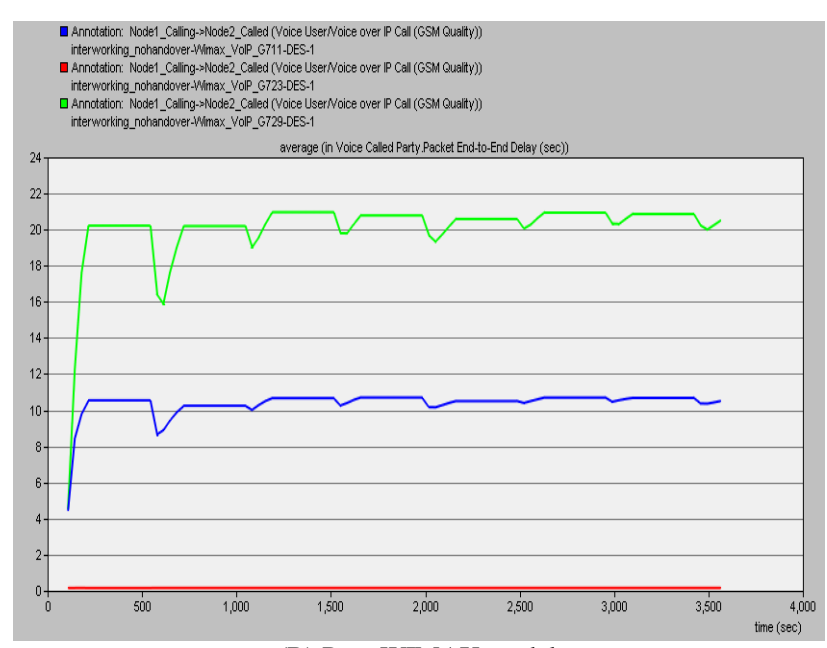

(B) Pure WIMAX model

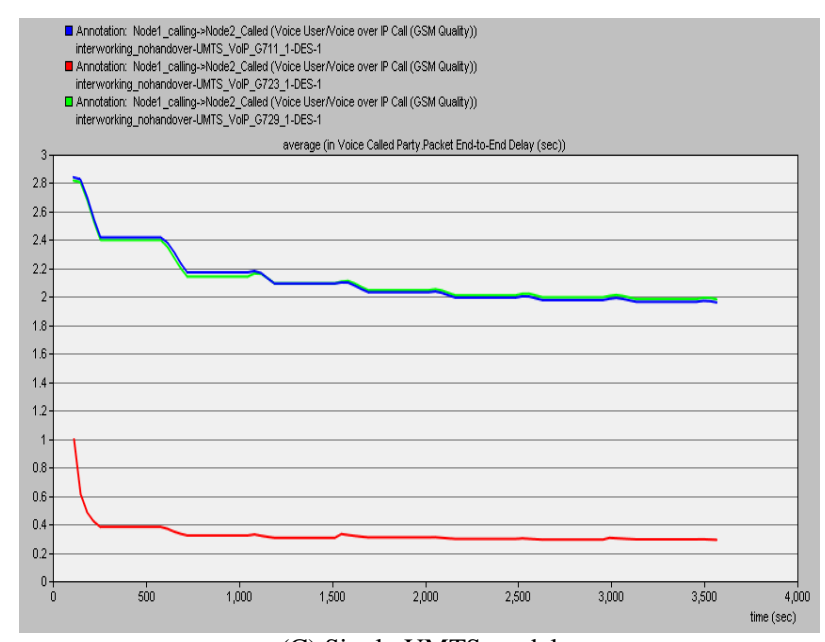

(C) Single-UMTS model

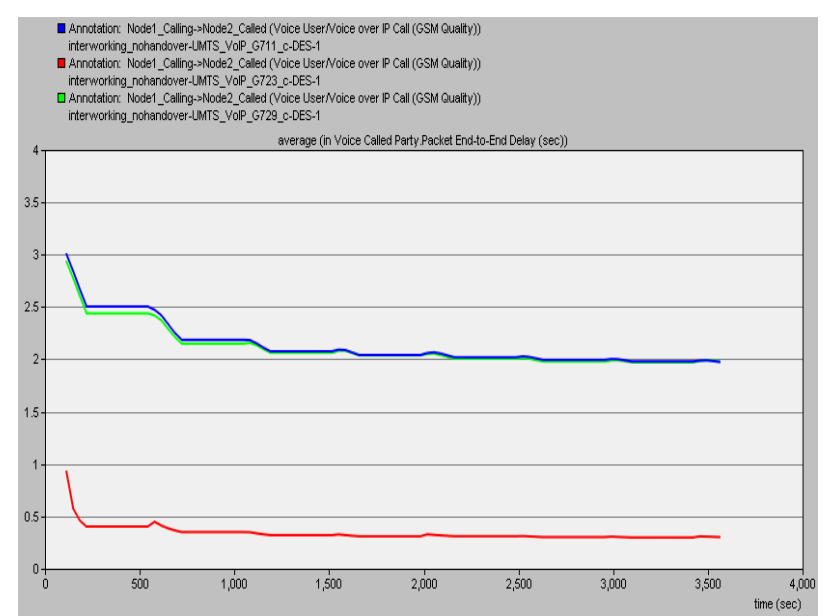

(D) Dual-UMTS model

Fig.8. Mean end-to-end delay results of the four models

Observing the arrival time of the packets in the called nodes shows a significant difference between the models. The amount of delay in both UMTS models is nearly the same and low which reaches to $3 \mathrm{~s}$ at most. However, for both heterogonous and pure WiMAX model the delay is increased highly to at least seven times more. Lower delay in UMTS models and very high delay in WiMAX model will explain that the high delay in the heterogonous model is related to merging the WIMAX radio access.

Thus, interworking of the WiMAX and UMTS can increase the delay in the UMTS networks. Considering the fact that real time traffics tend to be quite sensitive to delay and delay variations, in such cases interconnecting WiMAX and UMTS may not be desirable to end users or voice providers companies. The results also describe that the least delay belongs to G.723 codec in the four models.

We were curious that since connecting the WIMAX radio access mode to the UMTS increases the delay, what would be the side effect on the mean opinion score (MOS) of the voice packets. Thus we run the interworking model again to measure the MOS which is expressed in Fig. 9.

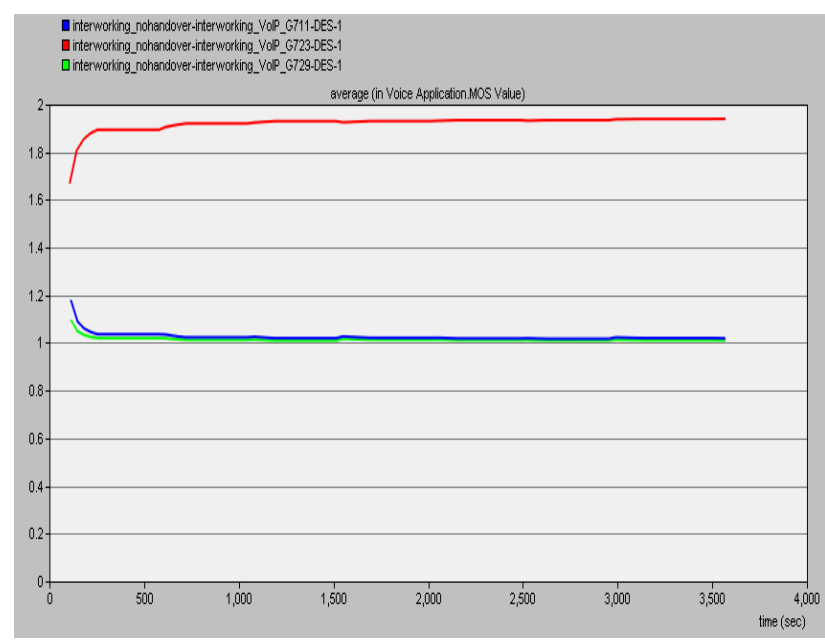

Fig.9. MOS in the interworking model 
The MOS values presented in Table 1 are considered as metric to evaluate the level of call quality.

Table 1. Voice Quality Levels For VoIP

\begin{tabular}{|l|l|}
\hline 5 & Excellent \\
\hline 4 & Good \\
\hline 3 & Fair \\
\hline 2 & Poor \\
\hline 1 & Bad \\
\hline
\end{tabular}

We notice from the interworking results in Fig. 9 that the MOS value is 2 which is considered poor voice perception quality. These results confirm our expectations as delay of the voice packets is highly relevant to achieved MOS; the higher the delay the more it degrades the quality of the calls. Thus, while pure UMTS networks offer a good level of call quality, their interworking with WiMAX will result in suffering low calls quality by the end users.

\section{Delay variations of audio streams}

To further demonstrate the benefits or constraints of the models in the proposed framework, the behavior of delay variants is studied by observing the simulated results provided for each model. The variations in delay introduced by internal structure of the heterogonous, pure WIMAX, UMTS-single, and UMTS-dual models are depicted in Fig. 10 A-D respectively.

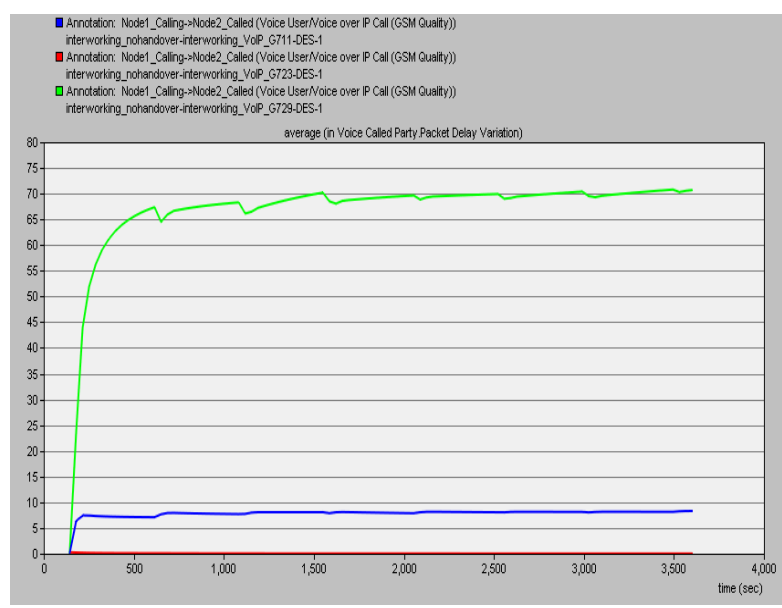

(A) Heterogonous model

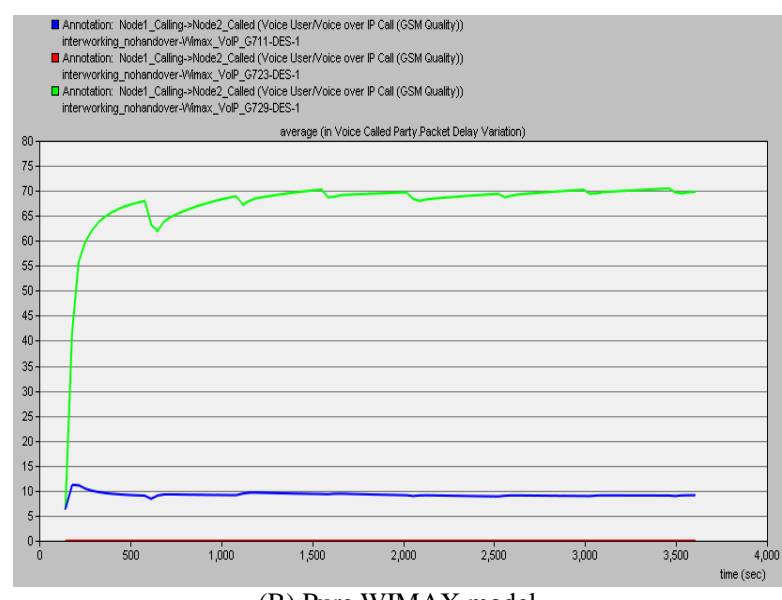

(B) Pure WIMAX model

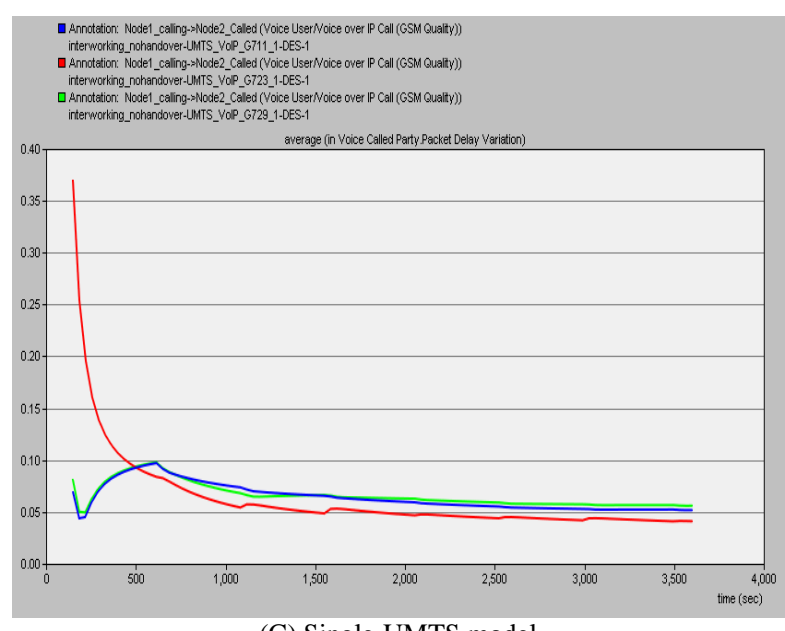

(C) Single-UMTS model

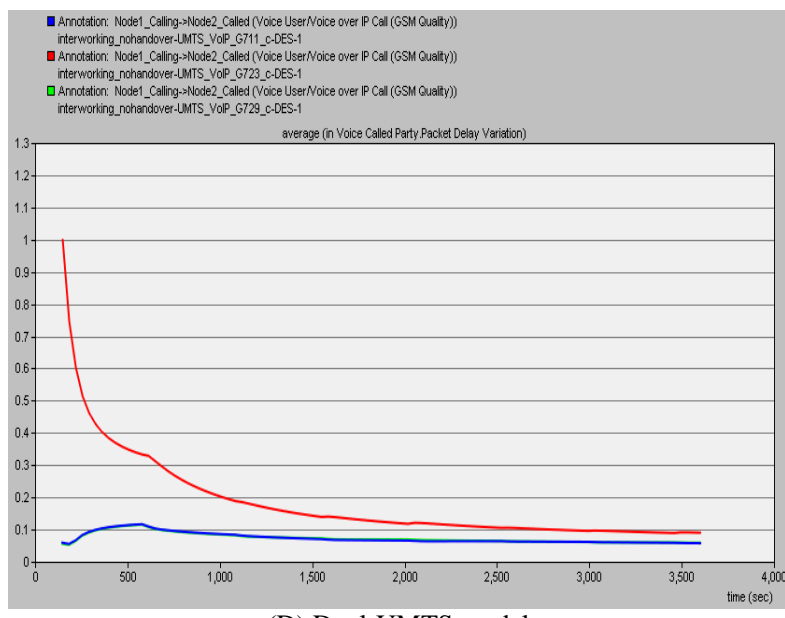

(D) Dual-UMTS model

Fig.10. Delay variations results of the four models

The analysis of delay variations results show that the delay variations reach the highest values in the pure WiMAX model while it is the lowest in the both UMTS models. Consequently, the high delay variations in WiMAX model directly affect the interworking model by highly increasing the variations in delay. Also comparing the two UMTS models shows higher delay variation when the UMTS design include two independent cores one for the calling party and the other for the called party. Thus, to get the best performance of the UMTS networks in term of delay variations it is better to have both end call parties in the same core to avoid propagation delay of the core network components. Also based on the results, avoiding G.729 codec in WiMAX networks can significantly improve the delay variations of the voice packets.

\section{CONCLUSION}

In this work, we presented a framework consists of four separate cellular network models for developing new techniques in demand for higher data rates particularly for cellular interworking. From the simulation results, it is concluded that while the models provide similar data rate, the pure UMTS models will achieve greater QoS 
performance for the call services in terms of less delay and variations in delay. Due to complexity of the internal structure of the WiMAX core, the end users experience poor call quality in these networks and any other network that is merged with them. Additionally, due to high compression rate of $\mathrm{G} .723$ codec, applying this codec to compress and decompress the voice packets in cellular based networks is not recommended especially when there are bandwidth limitation conditions as it leads to highly degradation of the network performance.

\section{REFERENCES}

[1] G.Vijayalakshmy and G. Sivaradje, WiMAX-UMTS Converging Architecture with IMS Signaling analysis to achieve QoS, Elsevier 2nd International Conference on Communication, Computing \& Security (CCCS-2012), 2012.

[2] I.I. Mohamed, A.A. Hadi, R. Othman, A. Oudah, Performance Analysis of Seamless Vertical Handover in $4 G$ Networks, Journal of Theoretical and Applied Information Technology, Vol. 79. No. 2, 2015.

[3] A.L. Karthika, M.G. Sumithra, and A.Shanmugam, Performance of voice in integrated WiMAX-WLAN and UMTS-WLAN, International Journal of Innovative Research in Science, Engineering and Technology. Vol. 2, No. 4, 2013.

[4] V. Bharathi and L. Nithyanandan, Efficient Cooperative Relaying in UMTS-WiMAX-WLAN Overlaid Heterogeneous Networks, Elsevier Processing of International Conference on Advances in Communication, Network, and Computing, CNC, 2014.

[5] P. Mehta and S. Baghla, Performance Evaluation of Heterogeneous Networks for Various Applications Using OPNET Modeler, International Journal on Recent and Innovation Trends in Computing and Communication, Vol. 3, No. 6, 2015.

[6] O. Arafat, M.A. Gregory, and M.M.A. Khan, Interworking Architecture between 3GPP IMS, Mobile IP and WiMAX in OPNET, IEEE 2nd International Conference on Electrical, Electronics and System Engineering (ICEESE), 2014.

[7] E. Kalaiselvi, S. Kokila, and G. Sivaradje, A Novel Resource Provisioning Algorithm for the Integrated UMBWiMAX-WLAN Overlay Networks. International Journal on Applications in Information and Communication Engineering Vol. 1, No. 12, 2015.
[8] O. Khattab and O. Alani, An Overview of Interworking Architectures in Heterogeneous Wireless Networks: Objectives, Features and Challenges, Proceedings of the Tenth International Network Conference (INC2014), 2014.

[9] A.A. Atayero and E.I. Adegoke, Interworking Architectures in Heterogeneous Wireless Networks: An Algorithmic Overview. International Journal of Computer Applications, Vol. 48, No .9, 2012.

[10] R.A. Hamada, S.A. Hanaa and M.I. Abdalla, SIP-Based Mobility Management for LTE-WiMAX-WLAN Interworking Using IMS Architecture. International Journal of Computer Networks (IJCN), Vol. 6, No. 1, 2014

[11] O. Khattab, Improving Initiation Phase for Vertical Handover in Heterogeneous Mobile Networks, International Journal of Engineering Trends and Technology (IJETT), Vol. 29, No. 3, 2015.

[12] G. Vijayalakshmy and G. Sivaradje, Loosely Coupled Heterogeneous Networks Convergence using IMS-SIP$A A A$, International Journal of Computer Applications, Vol. 85, No. 16, 2014.

[13] B.S.K Reddya and B. Lakshmib, BER Analysis with Adaptive Modulation Coding in MIMO-OFDM for WiMAX using GNU Radio, I.J. Wireless and Microwave Technologies, Vol.4, 2014.

[14] A.A. Subail, M. Ahsan, and M. Enayetullah, Development of A New Efficient Routing Scheme for WiMAX Mesh Networks, I. J. Modern Education and Computer Science, Vol.10, 2013.

[15] K. Jakimoski and T. Janevski, Priority Based Uplink Scheduling Scheme for WiMAX Service Classes, I.J. Information Technology and Computer Science, Vol. 8, 2013.

\section{Authors' Profiles}

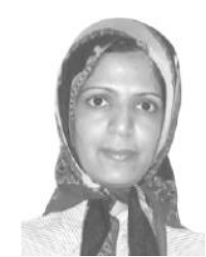

Mina Malekzadeh is an assistant professor and lecturer in the department of computer science at Hakim Sabzevari University. Her research interests include communication networks, network security, VoIP, and system development programming. She holds a Doctoral degree in computer security from UPM, MSc in software engineering from UPM, BSc in computer engineering from SBU.

How to cite this paper: Mina Malekzadeh,"Heterogeneous Cellular Interworking of WIMAX and UMTS for Sensitive Real-Time Services", International Journal of Information Engineering and Electronic Business(IJIEEB), Vol.8, No.6, pp.1-8, 2016. DOI: 10.5815/ijieeb.2016.06.01 